\title{
Quantum-to-classical crossover of quasi-bound states in open quantum systems
}

\author{
Henning Schomerus \\ Max-Planck-Institut für Physik komplexer Systeme, Nöthnitzer Straße 38, 01187 Dresden, Germany \\ Jakub Tworzydło \\ Instituut-Lorentz, Universiteit Leiden, P.O. Box 9506, 2300 RA Leiden, The Netherlands and \\ Institute of Theoretical Physics, Warsaw University, Hoża 69, 00-681 Warsaw, Poland
}

(Dated: September 23, 2018)

\begin{abstract}
In the semiclassical limit of open ballistic quantum systems, we demonstrate the emergence of instantaneous decay modes guided by classical escape faster than the Ehrenfest time. The decay time of the associated quasibound states is smaller than the classical time of flight. The remaining long-lived quasi-bound states obey random-matrix statistics, renormalized in compliance with the recently proposed fractal Weyl law for open systems [W. T. Lu, S. Sridhar, and M. Zworski, Phys. Rev. Lett. 91, 154101 (2003)]. We validate our theory numerically for a model system, the open kicked rotator.
\end{abstract}

PACS numbers: 03.65.Sq, 05.45.Ac, 05.45.Mt, 05.60.Gg

Open quantum systems are described by non-self-adjoint operators and hence have different spectral properties than closed ones. Instead of an orthogonal set of stationary bound states at real energies one encounters mutually non-orthogonal quasi-bound states $\psi_{n}$ with complex energies $E_{n}$, which decay exponentially in time with a uniform decay rate $\gamma_{n} / 2=$ $-\operatorname{Im} E_{n}$. The complex energies define the poles of the scattering matrix, for which the random-matrix theory (RMT) provides a universal benchmark in the case of random wave dynamics [1] (for applications to the transport in electronic nanostructures see [2]). RMT has been derived for disordered systems [3], while its status for ballistic dynamics is unsettled. In this paper we investigate ballistic systems that remain open in the classical limit- the decay mechanism then corresponds to ballistic escape of point particles into the asymptotic scattering region. (This can be realized in clean nanostructures and in driven atoms, provided the escape does not require tunneling.) One goal is to uncover and quantify deviations from RMT. We focus on the case of classically chaotic systems (our methods are easily transferred to integrable or mixed dynamics).

We show that in approaching the classical limit of these ballistic systems, the quasi-bound states separate into two different classes. The classes are discriminated by the Ehrenfest time $\tau_{\text {Ehr }}=\frac{1}{\lambda} \ln N$, up to which the escape observes quantum-to-classical correspondence [4]. ( $N$ is the number of ballistic escape channels and $\lambda$ is of the order of the Lyapunov exponent.) Phase space regions with classical escape faster than $\tau_{\text {Ehr }}$ support instantaneous ballistic decay with rate $\gamma_{n}$ larger than the inverse time of flight through the system. The quasi-bound states with instantaneous decay develop a large degree of degeneracy, which is accompanied by a drastic departure from orthogonality - the states are almost-linearly dependent. Non-orthogonality of states has been identified [5, 6] as the principal obstacle for the formulation of Weyl laws to rigorously count the states in open systems. We restore the applicability of the Weyl law and estimate the relative fraction of instantaneous decay modes as $1-\exp \left(-\tau_{\text {Ehr }} / \tau_{\text {dwell }}\right)$, where $\tau_{\text {dwell }}$ is the mean dwell time. The overwhelming majority of long-lived quasi-bound states associated to phase space regions with classical escape slower than $\tau_{\mathrm{Ehr}}$ are amenable for an effective RMT [7], in which the dependence of the effective matrix dimension on Planck's constant conforms with the recently discovered fractal Weyl laws in classically chaotic open systems [6].

The motivation for our work arises from the up-surging interest in the quantum-to-classical correspondence of open quantum systems, witnessed in particular over the past two years. That quantum-to-classical correspondence invalidates the assumption of wave-chaotic motion and implies nonuniversal corrections RMT was pointed out some while ago [8]. However, only recent experiments and numerical investigations could access the very large systems needed in view of the just-logarithmic scaling of $\tau_{\text {Ehr }}$ with the effective system size (here given by $N$ ). In stationary transport, the Ehrenfest time manifests itself in the systematic suppression of shot noise [9, 10, 11] and in the on-set of classical conductance fluctuations [12]. It also governs the impact of decoherence on dynamical systems coupled to heat-bath environments (open systems of a different kind than investigated here) [13, [14]. Spectral footprints of the Ehrenfest time have only been addressed for closed systems, in terms of the form factor of the spectral correlation function [8, 15] and the proximityinduced excitation gap in a normal conductor next to a superconductor [16, 17].

For simplicity, we formulate our theory of quasi-bound states for quantum maps $F$ with one degree of freedom, operating on states $\varphi$ in a Hilbert space of finite dimension $M[18]$. This dimension serves as the inverse of the effective Planck's constant, $h_{\text {eff }}=1 / M$. The evolution over one unit time step including loss is given by $\varphi(t+1)=Q F \varphi(t)$, where $Q$ is a projection operator of rank $M-N$ which describes survival in the system. This projection operator introduces sub-unitarity into the time evolution, which is the equivalent to non-selfadjointness in the energy domain. The mean dwell time is $\tau_{\text {dwell }}=M / N$. 

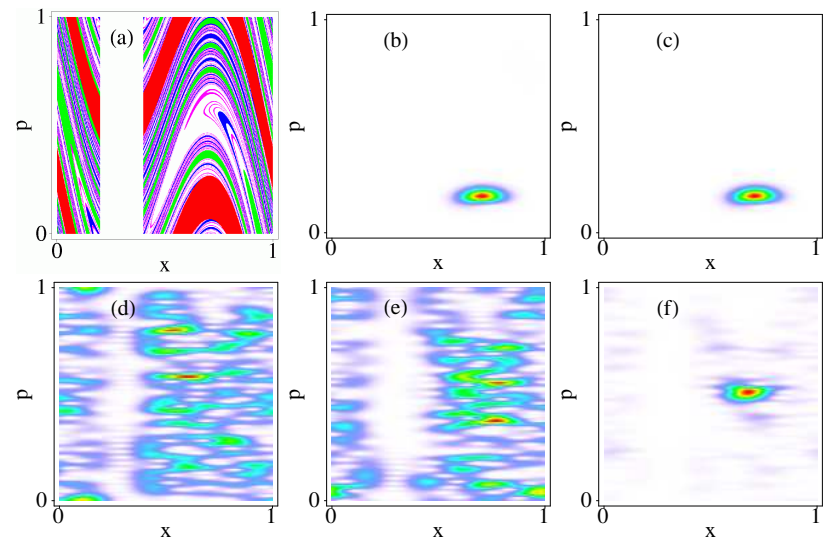

FIG. 1: (Color online) (a) Regions of escape after one (red), two (green), three (blue), four (magenta) iterations in the open standard map (9) with $K=7.5$ and escape for $x \in(0.2,0.4)\left(\tau_{\text {dwell }}=5\right)$. The other panels show Husimi representations of quasi-bound states for Hilbert space dimension $M=160$. (b,c) Short-lived states with instantaneous ballistic decay $\left(|\mu|<10^{-8}\right)$, localized on the classical pre-image of the opening. (d,e) Long-lived states with random-wave characteristics. (f) Trapped long-lived state (only one state of this kind exists for $M=160$; it has the smallest decay rate).

For $M \rightarrow \infty$ (i.e., $h_{\text {eff }} \rightarrow 0$ ) and fixed $\tau_{\text {dwell }}$, the system attains its classical limit, in which it is described by an areapreserving map $\mathcal{F}$ operating on a bounded two-dimensional phase space of normalized area 1 , while survival is described by a projection operator $\mathcal{Q}$ onto the complement of the openings. For illustration see the phase space portrait of the open classical standard map in Fig. 11a) (details of this system are given below).

Quasi-bound states are defined by the condition of quasistationarity

$$
Q F Q \psi_{n}=\mu_{n} \psi_{n}, \quad \mu_{n}=\exp \left(-i E_{n}\right),
$$

of the internal part of the wave function. Since $Q F Q$ is a sub-unitary operator, the eigenvalues have modulus $\left|\mu_{n}\right| \leq 1$, (i.e., lie inside the unit circle in the complex plane), and the quasi-energies $E_{n}$ have a negative imaginary part. The decay rate of a state $\psi_{n}$ is given by $\gamma_{n}=-2 \operatorname{Im} E_{n}$.

Our objective is to identify and count instantaneous decay modes by exploring the strict quantum-to-classical correspondence observed for escape times shorter than the Ehrenfest time. At the outset, note the set of $N$ trivial short-lived states in the kernel of $Q$, all of which have eigenvalue $\mu_{m}=0$ $(m=1, \ldots, N)$. They can be collected into the rows of an $M \times N$-dimensional matrix $P_{0}$, which fulfills $Q=\mathbb{1}-P_{0} P_{0}^{T}$. The basic building blocks for the construction of a much larger number of nontrivial short-lived states are the connected phase-space regions $A_{t, i}$ of escape after $t$ iterations $(t=1,2,3, \ldots)$, depicted in Fig. 11 a). We denote the union of all regions with fixed $t$ by $\mathcal{A}_{t}=\cup_{i \in I_{t}} A_{t, i}$, where $I_{t}$ contains all applicable indices. Formally associating a region $\mathcal{A}_{0}$ to the opening of the system, these regions partition phase space.

Next, we introduce the regions $\mathcal{B}_{t}=\cup_{i \in I_{t}^{\prime}} A_{t, i}$, where $I_{t}^{\prime}$ restricts the index to areas larger than a Planck cell $h_{\text {eff. In }}$ this part of phase space the escape of initial wave packets corresponds to the classical particle dynamics. Consequently, we call $\mathcal{B}=\cup_{t<\tau_{\mathrm{Ehr}}} \mathcal{B}_{t}$ the region of quantum-to-classical correspondence. The maximal index $\tau_{\mathrm{Ehr}}$ arises because of the finite size of the Planck cell, and defines the Ehrenfest time of quantum ballistic escape.

In order to construct states supported by the region $\mathcal{B}$ of quantum-to-classical correspondence, let us introduce the characteristic projection operators [19]

$$
\mathcal{P}_{t}=\int_{D\left(\mathcal{B}_{t}\right)} d x d p|\chi(x, p)\rangle\langle\chi(x, p)|
$$

where $|\chi(x, p)\rangle$ are minimal-uncertainty wave packets localized at position $x$ and momentum $p$, and $D(\cdot)$ denotes the characteristic function of a region. In the strict limit $h_{\mathrm{eff}} \rightarrow 0$, the operator $\mathcal{P}_{t}$ represents the characteristic function of the region $\mathcal{A}_{t}$. For finite $h_{\text {eff }}$, only the regions $\mathcal{B}_{t}$ are well-resolved by the wave packets. The operators $\mathcal{P}_{t}$ are defined with these smaller regions since this guarantees idempotency up to small corrections due to leakage. Hence, each $\mathcal{P}_{t}$ projects onto some subspace $H_{t}$ of a dimension $M_{t}=\operatorname{dim} H_{t}$. This implies the representation $\mathcal{P}_{t}=P_{t} P_{t}^{T}$, where the $M$-dimensional columns of $P_{t}$ are mutually orthogonal, $\mathcal{P}_{t} P_{t^{\prime}}=\delta_{t t^{\prime}} P_{t}$. We also introduce the complementary projector $\overline{\mathcal{P}}=1-\sum_{t} \mathcal{P}_{t}=$ $\bar{P} \bar{P}^{T}$.

Due to quantum-to-classical correspondence in the regions $\mathcal{B}_{t}$, the semiclassical dynamics propagates states from subspace $H_{t}$ to $H_{t-1}$ and finally to the opening, where they are destroyed by $Q$ :

$$
\begin{array}{ll}
Q F Q H_{t} \subset H_{t-1} & (t>1) ; \\
Q F Q H_{t}=\{0\} & (t=0,1) .
\end{array}
$$

Equation (3b) immediately exposes the $M_{0}+M_{1}$ states in $H_{0}$ and $H_{1}$ as quasi-bound states with eigenvalue $\mu_{n}=0$ (corresponding to instantaneous decay, $\gamma \rightarrow \infty$ ). Does this exhaust all short-lived states? In view of degeneracy of the associated eigenvalue and the sub-unitarity of $Q F Q$, this is by no means guaranteed. Indeed, Eqs. (3) naturally lead to a partial Schur decomposition [20],

$$
Q F Q \approx U T U^{\dagger}, \quad\left\{\begin{array}{l}
U=\left(P_{0}, P_{1}, \ldots, P_{\tau_{\mathrm{Ehr}}}, \bar{P}\right) \\
T=\left(\begin{array}{cc}
T_{11} & T_{12} \\
0 & T_{22}
\end{array}\right)
\end{array}\right.
$$

which reveals a much larger number of small eigenvalues. The unitary matrix $U$ leaves the eigenvalues invariant. The structure of the blocks of $T$ is obtained by considering the operation of $Q F Q$ on the columns in $P_{t}$. Equation (3) implies that $T_{11}$ is composed of sub-blocks that connect each subspace $H_{t}$ to $H_{t-1}$ (but not in the opposite direction). Hence, this matrix is upper triangular and all the diagonal elements vanish. It follows that the eigenvalue $\mu_{n}=0$ indeed has an algebraic multiplicity [20] $M_{\mathrm{Ehr}}=\sum_{t<\tau_{\mathrm{Ehr}}} M_{t}$. 
The difference between $M_{\mathrm{Ehr}}$ and $M_{0}+M_{1}$ can be traced back to the break-down of the conditions for the conventional Weyl law known for closed systems [21], which assumes an orthogonal set of eigenvectors and translates this into a uniform covering of phase space. Perturbation theory shows that the small leakage out of the subspaces in Eqs. (3) lifts the degeneracy of eigenvalues $\mu_{n} \approx 0$, but the resulting $M_{\text {Ehr }}$ eigenvectors are almost-linearly dependent, with their major component confined to the subspaces $H_{0}$ and $H_{1}$. The conventional Weyl law would underestimate their number as $M_{0}+M_{1} \approx M\left(\left|\mathcal{B}_{0}\right|+\left|\mathcal{B}_{1}\right|\right)$. The Schur decomposition carefully accounts for the admixture of the other subspaces $H_{t}$ with $t \geq 2$, and provides an orthonormalized basis $\left(P_{0}, P_{1}, \ldots, P_{\tau_{\mathrm{Ehr}}}\right)$ for the short-lived states. Equipped with such a basis, we now can formulate a new Weyl law in analogy to the conventional case of a closed systems: The rank $M_{t}$ of the characteristic projectors $\mathcal{P}_{t}$ can be estimated by the area covered by the regions $\mathcal{B}_{t}, M_{t} \approx M\left|\mathcal{B}_{t}\right|$. A direct consequence is the estimate $M_{\mathrm{Ehr}} \approx M|\mathcal{B}|$ for the number of short-lived states.

Up to this point, our construction applies independent of the nature of the classical dynamics, which may be integrable, mixed, or chaotic. From now on we focus on classically chaotic systems, which show a minimal amount of system-specific details provided the dynamics are 'sufficiently ergodic' $\left(\tau_{\text {dwell }} \lambda \gg 1\right)$. In parallel to the considerations in Ref. [7, 11], a typical area $\left\langle\left|A_{t, i}\right|\right\rangle \approx \tau_{\text {dwell }}^{-1} \exp (-t \lambda)$ shrinks exponentially with time $t$ and equates to the Planck cell $h_{\text {eff }}=1 / M$ at $\tau_{\text {Ehr }}=\frac{1}{\lambda} \ln N$. The number of individual regions in $\mathcal{B}_{t}$ at given $t$ is found by balancing the exponential shrinking of areas with the survival probability $P(t) \sim \tau_{\text {dwell }}^{-1} \exp \left(-t / \tau_{\text {dwell }}\right)$ for uniformly sampled starting points in phase space. The probability density $P(|A|)$ to initially reside in a region of area $|A|$ then takes the power law

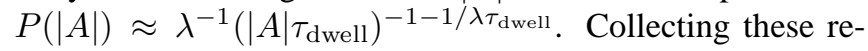
sults, the region of quantum-to-classical correspondence covers an area $|\mathcal{B}| \approx 1-\exp \left(-\tau_{\text {Ehr }} / \tau_{\text {dwell }}\right)$. We arrive at the estimate

$$
M_{\mathrm{Ehr}} \approx M\left[1-\exp \left(-\tau_{\mathrm{Ehr}} / \tau_{\mathrm{dwell}}\right)\right]
$$

of the number of instantaneous decay modes in a classically chaotic system.

The complementary region $\overline{\mathcal{B}}$ covers an area

$$
|\overline{\mathcal{B}}| \approx \exp \left(-\tau_{\text {Ehr }} / \tau_{\text {dwell }}\right)=N^{-1 / \lambda \tau_{\text {dwell }}} .
$$

In this region we can expect fully developed wave chaos, described by RMT with an effective matrix dimension

$$
\bar{M}=M-M_{\mathrm{Ehr}} \approx M N^{-1 / \lambda \tau_{\mathrm{dwell}}} .
$$

Finally, with the RMT prediction of Ref. [22], the number of decay modes with $\gamma_{n}$ smaller than a fixed value $\gamma<1 / \tau_{\text {Ehr }}$ is estimated as

$$
n_{\gamma}=\bar{M}\left[1-\tau_{\text {dwell }}^{-1}\left(1-e^{-\gamma}\right)^{-1}\right] .
$$

For fixed $\tau_{\text {dwell }}$ we obtain $n_{\gamma} \propto h_{\text {eff }}^{d}$, hence, a power-law dependence on $h_{\mathrm{eff}}=1 / M$ with non-integer exponent $d=$ $\left(1 / \lambda \tau_{\text {dwell }}\right)-1$. This is precisely of the form of the fractal Weyl laws recently put forward by Liu et al. [6], who formulated a trace formula for the long-lived quasi-bound states associated to the classical repeller of a chaotic open system (while we arrived at the result by constructing the short-lived states).

We examine our predictions for a representative system with ballistic openings, the open kicked rotator also used in previous investigations [11, 12, 16] of the Ehrenfest time. In the classical limit, the phase space of this system is the torus $(x, p) \in[0,1) \otimes[0,1)$ with periodic boundary conditions. The phase-space density $\rho_{t+1}(x, p)=\mathcal{Q F}\left(\rho_{t}(x, p)\right)$ evolves in discrete time-steps according to the standard map $\mathcal{F}$, specified by

$$
\begin{aligned}
& x_{t+1}=x_{t}+p_{t}+\frac{K}{4 \pi} \sin 2 \pi x_{t} \quad(\bmod 1), \\
& p_{t+1}=p_{t}+\frac{K}{4 \pi}\left(\sin 2 \pi x_{t}+\sin 2 \pi x_{t+1}\right) \quad(\bmod 1),
\end{aligned}
$$

which is followed by leakage in terms of a projection operator $\mathcal{Q}$ that discards the density in a coordinate strip $(x, p) \in$ $\mathcal{A}_{0}=\left(x_{0}, x_{0}+1 / \tau_{\text {dwell }}\right) \otimes[0,1)$. For kicking strength $K \gtrsim 7$, the standard map displays well-developed global chaos, characterized by a Lyapunov exponent $\lambda \approx \ln (K / 2)$. We fix $K=7.5, x_{0}=0.2, \tau_{\text {dwell }}=5$. The quantum dynamics takes place in a Hilbert space of even integer dimension $M$, spanned by a basis of discretized position states at $x=m / M$, $m=0,1, \ldots, M-1$. The time-evolution operator is

$$
F_{m m^{\prime}}=\frac{1}{\sqrt{i M}} e^{\frac{i \pi}{M}\left(m^{\prime}-m\right)^{2}-\frac{i M K}{4 \pi}\left[\cos \frac{2 \pi m}{M}+\cos \frac{2 \pi m^{\prime}}{M}\right]},
$$

and $Q=\operatorname{diag}\left(\mathbb{1}_{N \times N}, O_{N \times N}, \mathbb{1}_{(M-2 N) \times(M-2 N)}\right)$.

Husimi phase-space representations $H(x, p)=$ $\left|\left\langle\chi(x, p) \mid \psi_{n}\right\rangle\right|^{2}$ of representative quasi-bound states for $M=160$ are shown in Fig. 1 b-f). The short-lived states in panels $(\mathrm{b}, \mathrm{c})$ have major component in the space $H_{1}$ associated to the region $\mathcal{A}_{1}$, and overlap in violation of the assumptions for the conventional Weyl law. Long-lived states $(d, e)$ show random, delocalized wave patterns. Exceptions are a small number of long-lived trapped states, which are localized in the region of slowest escape. For $M=160$ only one such state exists, which is shown in Fig. 10 f).

Figure 2 a) shows the decay factors $\left|\mu_{n}\right|=\exp \left(-\gamma_{n} / 2\right)$, ordered by the decay rate $\gamma_{n}$ (starting with the long-lived states). The data covers a range of inverse Planck's constants between $M=80$ and $M=20480$, and is presented as a function of the relative index $n / M$. In accordance with the increasing area covered by the region $\mathcal{B}$ of quantum-to-classical correspondence, the relative fraction of short-lived states increases with $M$. Figure 2 (b) shows the decay factors as a function of the renormalized relative index $n / \bar{M}$, with $\bar{M}$ estimated from Eq. (7) and $\lambda=1.32$ approximated by the Lyapunov exponent of the closed system. The collapse onto one 

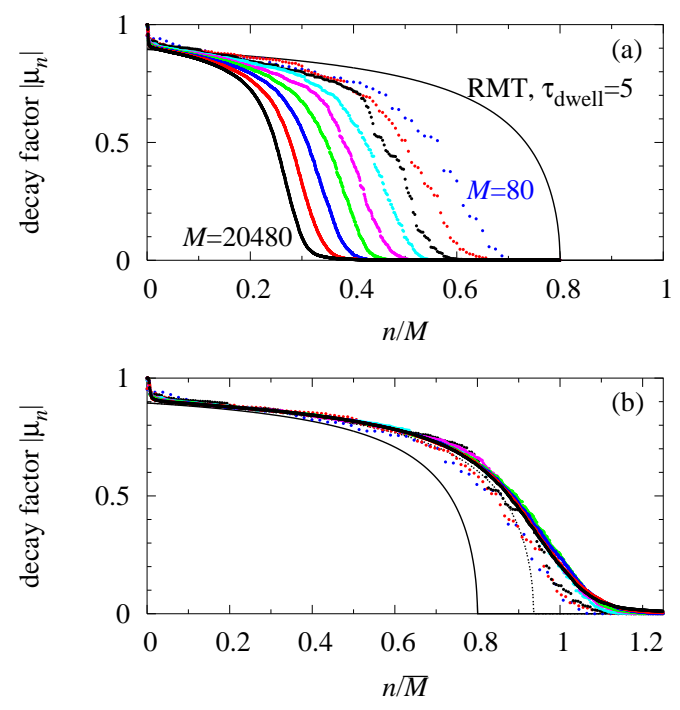

FIG. 2: (Color online) Ordered decay factors $\left|\mu_{n}\right|=\exp \left(-\gamma_{n} / 2\right)$ of the open kicked rotator with $M=2^{m} \cdot 80, m=0, \ldots, 8$, and the other parameters as in Fig. 11a), plotted as a function of the relative index $n / M$ (panel a) or of the renormalized relative index $n / \bar{M}$, where $\bar{M}$ is obtained from Eq. (7) (panel b). The solid curve is the RMT result [22]. The dashed curve is this result with $\bar{M}$ fitted to the data.

curve for all values of $M$ confirms the predicted scaling (5) of the number of short-lived states with the Ehrenfest time.

Also shown in Fig. 22 is the result of RMT, obtained by substituting the matrix $F$ with a random matrix from the circular orthogonal ensemble. The almost perfect agreement found by fitting the effective matrix dimension $\bar{M}$ to the data [dashed curve in Fig. 2]b)] demonstrates that effective RMT applies to a vast majority of the long-lived states. Since the number of states $n_{\gamma}$ is obtained by inverting this curves, the collapse of the data onto the effective RMT curve confirms the fractal Weyl law 8 .

In summary, we identified instantaneous decay modes (exceptionally short-lived quasi-bound states) in open ballistic quantum systems, which capitalize on escape routes shorter than the Ehrenfest time. The large numbers in which these states emerge in the semiclassical limit is revealed only after a regularization of Weyl's law, which is required because of the almost-linear dependence of these states. For chaotic classical dynamics, the remaining long-lived states obey a RMT with an effective matrix dimension complying to a fractal Weyl law, from which one can conclude that such laws and the formation of instantaneous decay modes are intimately related.

We gratefully acknowledge enlightening discussions with
M. Zworski and S. Nonnenmacher. J.T. acknowledges the financial support provided through the European Community's Human Potential Programme under contract HPRNCT-2000-00144, Nanoscale Dynamics.

[1] Y. V. Fyodorov and H.-J. Sommers, J. Math. Phys. 38, 1918 (1997); JETP Lett. 72, 422 (2000).

[2] C. W. J. Beenakker, Rev. Mod. Phys. 69, 731 (1997).

[3] K. B. Efetov, Adv. Phys. 32, 53 (1983).

[4] G. M. Zaslavsky, Phys. Rep. 80, 157 (1981).

[5] J. Sjöstrand, Duke Math. J. 60, 1 (1990); K. Lin, J. Comput. Phys. 176, 295 (2002); K. Lin and M. Zworski, Chem. Phys. Lett. 355, 201 (2002).

[6] W. T. Lu, S. Sridhar, and M. Zworski, Phys. Rev. Lett. 91, 154101 (2003).

[7] P. G. Silvestrov, M. C. Goorden, and C. W. J. Beenakker, Phys. Rev. B 67, 241301(R) (2003).

[8] I. L. Aleiner and A. I. Larkin, Phys. Rev. B 54, 14423 (1996); Phys. Rev. E 55, R1243 (1997).

[9] O. Agam, I. Aleiner, and A. Larkin, Phys. Rev. Lett. 85, 3153 (2000).

[10] S. Oberholzer, E. V. Sukhorukov, and C. Schönenberger, Nature 415, 765 (2002).

[11] J. Tworzydło, A. Tajic, H. Schomerus, and C. W. J. Beenakker, Phys. Rev. B 68, 115313 (2003).

[12] J. Tworzydło, A. Tajic, and C. W. J. Beenakker, Phys. Rev. B 69, 165318 (2004); Ph. Jacquod and E. V. Sukhorukov, Phys. Rev. Lett. 92, 116801 (2004)

[13] I. Garcia-Mata, M. Saraceno, and M. E. Spina, Phys. Rev. Lett. 91, 064101 (2003).

[14] A. R. R. Carvalho, R. L. de Matos Filho, and L. Davidovich, Phys. Rev. E 70, 026211 (2004).

[15] C. Tian and A. I. Larkin, cond-mat/0310429 (2003).

[16] Ph. Jacquod, H. Schomerus, and C. W. J. Beenakker, Phys. Rev. Lett. 90, 207004 (2003).

[17] M. G. Vavilov and A. I. Larkin, Phys. Rev. B 67, 115335 (2003); P. G. Silvestrov, M. C. Goorden, and C. W. J. Beenakker, Phys. Rev. Lett. 90, 116801 (2003).

[18] Our results can be generalized by considering the Poincaré surface of section at the boundary of the scattering region (phase space area $A$, area occupied by the openings $A_{0}$ ). We identify $F \exp (i E)$ with the transfer operator [E. B. Bogomolny, Nonlinearity 5, 805 (1992)] and $Q$ with the projector onto the confinement [B. Georgeot and R. E. Prange, Phys. Rev. Lett. 74, 4110 (1995)]. For conserved wavenumber $k, M=k A$ and $N=k A_{0}$.

[19] For properties of such projectors see e.g. R. O. Vallejos and M. Saraceno, J. Phys. A 32, 7273 (1999).

[20] G. H. Golub and C. F. van Loan, Matrix Computations, 3rd ed. (Johns Hopkins University Press, Baltimore, 1996).

[21] J. Marklof and S. O'Keefe, preprint nlin.CD/0404038 (2004).

[22] K. Życzkowski and H.-J. Sommers, J. Phys. A: Math. Gen. 33, 2045 (2000). 